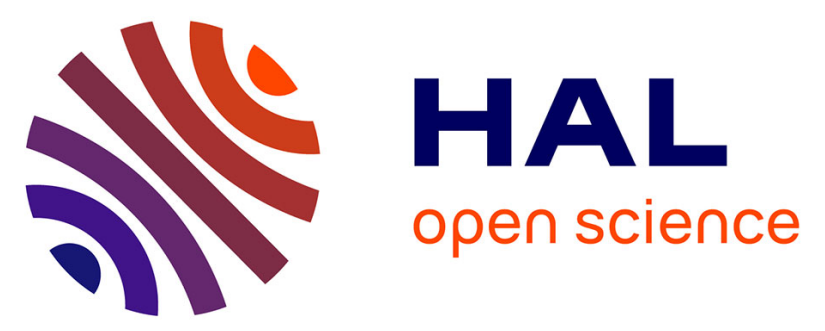

\title{
Aquaporin 1 (AQP1) expression is a novel characteristic feature of a particularly aggressive subgroup of basal-like breast carcinomas
}

\author{
Friedrich Otterbach, Rainer Callies, Michael Adamzik, Rainer Kimmig, \\ Winfried Siffert, Kurt W. Schmid, Agnes Bankfalvi
}

\section{To cite this version:}

Friedrich Otterbach, Rainer Callies, Michael Adamzik, Rainer Kimmig, Winfried Siffert, et al.. Aquaporin 1 (AQP1) expression is a novel characteristic feature of a particularly aggressive subgroup of basal-like breast carcinomas. Breast Cancer Research and Treatment, 2009, 120 (1), pp.67-76. 10.1007/s10549-009-0370-9 . hal-00535348

\author{
HAL Id: hal-00535348 \\ https://hal.science/hal-00535348
}

Submitted on 11 Nov 2010

HAL is a multi-disciplinary open access archive for the deposit and dissemination of scientific research documents, whether they are published or not. The documents may come from teaching and research institutions in France or abroad, or from public or private research centers.
L'archive ouverte pluridisciplinaire HAL, est destinée au dépôt et à la diffusion de documents scientifiques de niveau recherche, publiés ou non, émanant des établissements d'enseignement et de recherche français ou étrangers, des laboratoires publics ou privés. 


\title{
Aquaporin 1 (AQP1) expression is a novel characteristic feature of a particularly aggressive subgroup of basal-like breast carcinomas
}

\author{
Friedrich Otterbach $\cdot$ Rainer Callies $\cdot$ Michael Adamzik • \\ Rainer Kimmig · Winfried Siffert · Kurt W. Schmid • \\ Agnes Bankfalvi
}

Received: 11 June 2008/ Accepted: 5 March 2009/Published online: 21 March 2009

(C) Springer Science+Business Media, LLC. 2009

\begin{abstract}
Aquaporin1 (AQP1) is a water channel protein that facilitates water flux across cell membranes. It is widely expressed in epithelial and endothelial cells in several tissues. AQP1 is also associated with angiogenesis, cell migration and metastasis in some human malignancies. In this study the immunohistochemical expression of AQP1 in 203 invasive breast carcinomas with long-term follow up was investigated. AQP1 expression was demonstrated in 11 tumours (5.4\%) and showed highly significant correlation with high tumour grade, medullary-like histology, "triplenegativity", cytokeratin 14 and smooth muscle actin expression. In univariate analysis, AQP1 was significantly associated with poor prognosis. In multivariate analysis, AQP1 expression proved to be an independent prognostic marker if stratified by age, tumour size, lymph node status,
\end{abstract}

F. Otterbach $(\bowtie)$ K K. W. Schmid · A. Bankfalvi

Institute of Pathology and Neuropathology, University Hospital of Essen, University of Duisburg-Essen, Hufelandstraße 55, 45122 Essen, Germany

e-mail: friedrich.otterbach@uk-essen.de

R. Callies $\cdot$ R. Kimmig

Clinic of Obstetrics and Gynaecology, University Hospital

of Essen, University of Duisburg-Essen, Essen, Germany

M. Adamzik

Clinic of Anaesthesiology and Critical Care, University Hospital of Essen, University of Duisburg-Essen, Essen, Germany

W. Siffert

Institute of Pharmacogenetics, University Hospital of Essen, University of Duisburg-Essen, Essen, Germany

F. Otterbach · R. Kimmig - W. Siffert - K. W. Schmid

West German Cancer Centre Essen (WTZE), Essen, Germany

F. Otterbach · R. Callies · R. Kimmig - K. W. Schmid

University Breast Cancer Centre Essen, Essen, Germany histological grade, ER status and CMF therapy. Our results strongly suggest that AQP1 expression is a new characteristic feature of a particularly aggressive subgroup of basal-like breast carcinomas.

Keywords Aquaporin - AQP1 - Breast carcinoma . Prognosis

\section{Introduction}

Aquaporins (AQPs) belong to the major intrinsic protein (MIP) family of small, transmembrane, channel-forming glycoproteins that facilitate rapid water transport and, in the subgroup of aquaglyceroporins, the transport of small solutes such as glycerol, across biological membranes. Since the identification of AQP1 on the cell membranes of erythrocytes in 1988 by Agre and collegues [1], 13 members of the AQP family have been identified in humans to date. AQPs are expressed in many epithelia and endothelia in fluid transporting tissues, such as kidney tubules and exocrine gland epithelia, where their role is well understood. However, they are also highly expressed in cell types not directly involved in fluid fluxes, such as epidermis, fat-tissue, urinary bladder cells and astroglia [2, 3]. This has raised the question whether AQPs facilitate an additional function(s) other than water transport.

Recently the interest in the AQP family has increased, as several studies suggested a role for AQPs in various diseases [4]. AQP expression has been reported in a variety of human malignancies, e.g. tumours of the brain, prostate, breast, ovary, colon and lung. AQP expression has been proposed as a diagnostic and/or prognostic parameter [5]; The identification of AQP1 as an early response gene to mitogens [6] as well as the demonstration of AQP1 in 
tumour microvessels indicate that aquaporins may be involved both in tumour growth and angiogenesis. This is further supported by the finding of a reduced tumour growth and angiogenesis in AQP1-deficient mice [7]. Moreover, AQP1 expression could be associated with increased migration and metastatic potential of tumour cell in vitro, suggesting a novel function for AQP expression in high grade tumours [5].

The aim of the present study was to elucidate possible associations of AQP1 expression in breast carcinomas with tumour characteristics and survival of women suffering from sporadic breast cancer. For this purpose, AQP1 expression was immunohistochemically determined in a series of 203 well-characterised invasive breast carcinomas with long-term clinical follow-up. The data obtained were compared with a broad range of clinicopathological parameters, including overall survival of patients.

\section{Materials and methods}

\section{Patients and tumours}

The present study was based on a consecutive series of 203 cases of primary breast carcinomas with long-term clinical follow-up, presenting between 1989 and 1993, which were diagnosed at the Institute of Pathology and Neuropathology and operated with curative intent at the Clinic of Obstetrics and Gynaecology of the University Hospital of Essen, Germany. Patients were treated without exception in a uniform way according to standard protocols. Patients' clinical history and tumour characteristics were obtained from the pathology database. Tumour type, TNM-staging and grading were reassessed according to the WHO-Classification of Tumours of the Breast 2003 [8] and the 6th edition of the TNM Classification System 2002 [9]. Grading was performed according to the criteria of Elston and Ellis [10]. The Nottingham Prognostic Index (NPI) was calculated by using the following equation: $\mathrm{NPI}=0.2$ tumour size $(\mathrm{cm})+$ grade $(1-3)+$ lymph node score (1-3). Three prognostic groups were defined using the following cut-off score values: good $(<3.41)$, moderate (3.41-5.4) and poor (>5.4) [11].

In addition to histomorphology, immunophenotype profiles were also used for the definitive diagnosis of various subtypes of breast carcinoma. Basal-like carcinomas were primarily classified on the basis of their characteristic morphology and expression of the basal cell marker cytokeratin 14 (CK14) or smooth-muscle actin, irrespective of the expression of hormone receptors or HER2 in the first instance. Morphologically, most of these tumours revealed medullary-like histology including syncytial growth pattern, geographical necrosis, acellular areas, pronounced lymphoplasmacytic infiltrates, high mitotic count, high nuclear polymorphism and the absence of tubule formation [16]. The majority of tumours had a triple-negative immunophenotype (estrogen-receptor-negative, progesterone receptor-negative and HER-2 negative) as described by Laakso et al. [13], Banerjee et al. [14] and Rakha et al. [15], as well.

Survival data were obtained from the local municipal registry; the median follow-up period of patients still alive was 123 months (range 80-155 months). Patients with small carcinomas (pT1mic/pT1a), primary distant metastases, bilateral carcinomas, concurrent malignant tumours, or non-tumour related death were excluded from the study. Prognostic factors were analysed according to recent recommendations from McShane and co-workers [12] for tumour marker prognostic studies.

The study was strictly performed according to the Declaration of Helsinki and approved by the local Ethics Committee of the University Hospital of Essen.

Tissue microarray construction

Routinely formalin-fixed and paraffin-embedded tumour tissue blocks were retrieved from the files of the Institute of Pathology and Neuropathology (University Hospital of Essen, Germany) and processed using tissue microarray (TMA) technology. In short: one tumour tissue core with a diameter of $3 \mathrm{~mm}$ was extracted from each donor block using a skin biopsy punch (PFM, Cologne, Germany) and brought into recipient blocks $(n=14)$, each containing a maximum of 22 tumour samples. One tissue core of normal thyroid and liver tissue in preset positions in each block served as control tissue and helped with the orientation.

Immunohistochemistry and scoring

Sections of $5 \mu \mathrm{m}$ thickness of TMA were cut and mounted on SuperFrost ${ }^{\circledR}$ Plus slides (Menzel, Braunschweig, Germany). Following individually optimised heat-based antigen retrieval for each antibody, each set of 14 glass slides comprising the TMAs was immunostained with commercially available antibodies. The following antibodies were used: anti-ER [clone SP1, DCS (Hamburg, Germany), dilution 1:300, antigen retrieval: $30 \min 95^{\circ} \mathrm{C}$ water bath, citrate buffer, $\mathrm{pH}$ 6.0], anti-cytokeratin 14 (CK14) [clone LL002, DCS (Hamburg, Germany), dilution 1:400, antigen retrieval: $20 \mathrm{~min} 95^{\circ} \mathrm{C}$ water bath, citrate buffer, $\mathrm{pH}$ 6.0], anti-smooth muscle actin (SMA) [clone 1A4, DakoCytomation (Glostrup, Denmark), dilution 1:500, without antigen retrieval], anti-HER2/neu 
[HercepTest Kit K5207, DakoCytomation (Glostrup, Denmark), 40 min $95^{\circ} \mathrm{C}$ water bath in HercepTest buffer] and anti-AQP1 [clone B-11, Santa Cruz Biotechnology, Inc., Santa Cruz, CA, USA) dilution 1:1000, antigen retrieval: $30 \min 95^{\circ} \mathrm{C}$ water bath, citrate buffer, $\mathrm{pH}$ 6.0]. Automated immunohistochemistry was performed using the Dako Autostainer Plus System (DakoCytomation, Carpinteria, CA, USA) with the anti-mouse IgG EnVision Plus detection kit (DakoCytomation, Carpinteria, CA, USA) for secondary and tertiary immunoreactions. Reaction products were developed with diamino-benzidine (DAB), according to general protocols. Positive and negative control sections were included in each run, which showed appropriate results.

Stained sections were independently reviewed by FO and $\mathrm{AB}$ in a blind fashion, not knowing the results of any of the investigated tumour characteristics and survival data excluding the apparent histological tumour type and grade. Discordant cases were discussed using a multi-headed microscope until agreement was achieved. AQP1, ER, PR, CK14 and SMA reactions were scored positive, if $>10 \%$ of tumour cells showed any staining; for AQP1 only membranous, for ER expression only nuclear, for CK14 and SMA only cytoplasmic reactions were regarded as positive. HER2/neu expression was assessed according to the DAKO-score. A moderate or strong complete membranous staining of $>10 \%$ of tumour cells (DAKO-score $3+$ ) was interpreted as HER2/neu overexpression. "Triple-Negativity" was defined as the absence of ER and PR expression as well as HER2/neu overexpression. For statistical analysis, staining results were classified as negative or positive according to the respective cut-off values.

\section{Statistical analysis}

Statistical analysis was performed using SPSS 14.0 (SPSS, Chicago, USA). Descriptive statistics for continuous measures are given as the mean and/or median with minimum and maximum values and standard deviation, for discrete data frequency counts and percentages are tabulated. The Pearson's $\chi^{2}$-test and the Fisher exact test, if appropriate, were used to compare AQP1 expression with clinical and pathological findings. Correlation between parameters was analysed by the Pearson's pair-wise correlation matrix. The prognostic value of all parameters was studied in univariate analysis. Survival probabilities were estimated by the Kaplan-Meier method; differences assessed by the logrank test (Mantel-Cox method). Multivariate analysis was used to determine the independent prognostic value of selected variables using Cox's proportional hazards linear regression model with backward stepwise regression. All test were two-tailed with a confidence interval of $95 \%$, significance was defined at $p<0.05$.

\section{Results}

AQP1 expression in carcinomas and tumour-adjacent normal-appearing breast tissues

AQP1 expression was detected in 11 cases $(5.4 \%)$ of the present series of invasive breast carcinomas, all of them represented basal-like carcinomas with characteristic morphological and immunophenotypic features as described previously. In large tumour cells, immunostaining was predominantly membranous; however, in the majority of positive cases a less intense cytoplasmic staining could also be demonstrated (Fig. 1). In smaller immunoreactive tumour cells, membranous and cytoplasmic staining could not be distinctly distinguished by light microscopy. In some of the positive cases, the intensity of immunostaining was more pronounced at the tumour invasion front (Fig. 2). Adjacent to geographical necrosis, the AQP1 immunostaining was usually decreased. A strong AQP1 expression could be observed in the (myo-)fibroblastic cells of the tumour stroma in $21(10.3 \%)$ invasive carcinomas, the tumour cells of which were uniformly AQP1 negative. Interestingly the tumour stroma of AQP1 positive cases did not show any significant AQP1 expression. A weak staining of the specialized periductal or intralobular stroma was only noticed in single cases of postmenopausal breast tissue.

Adjacent to carcinomas, there was a strong membranous reaction with an admixture of cytoplasmic staining in all myoepithelial cells of normal lobules and ducts (Fig. 3), in endothelial cells of capillary and venous blood vessels (Fig. 4), smooth muscle cells and the perineural sheets of peripheral nerve fibres. Only a minor portion of luminal epithelial cells in normal lobules revealed strong AQP1 expression (Fig. 3). Occasionally, in areas with ductal

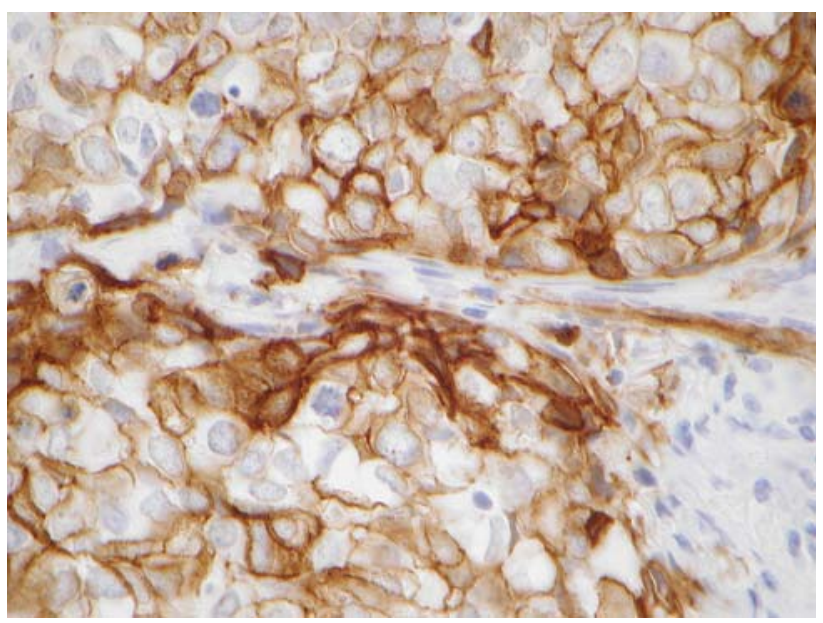

Fig. 1 AQP1 positive breast carcinoma with strong membranous staining 


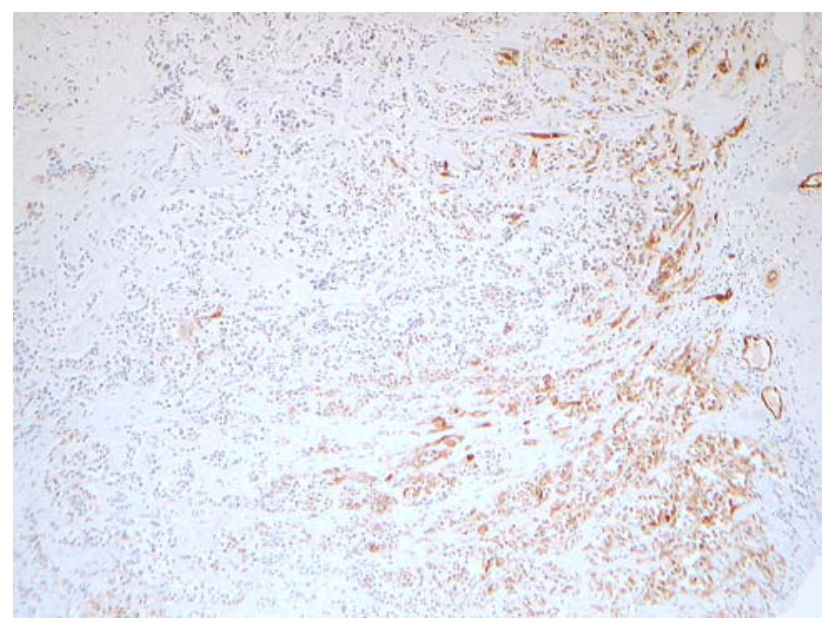

Fig. 2 Increased AQP1 staining at the tumour invasion front

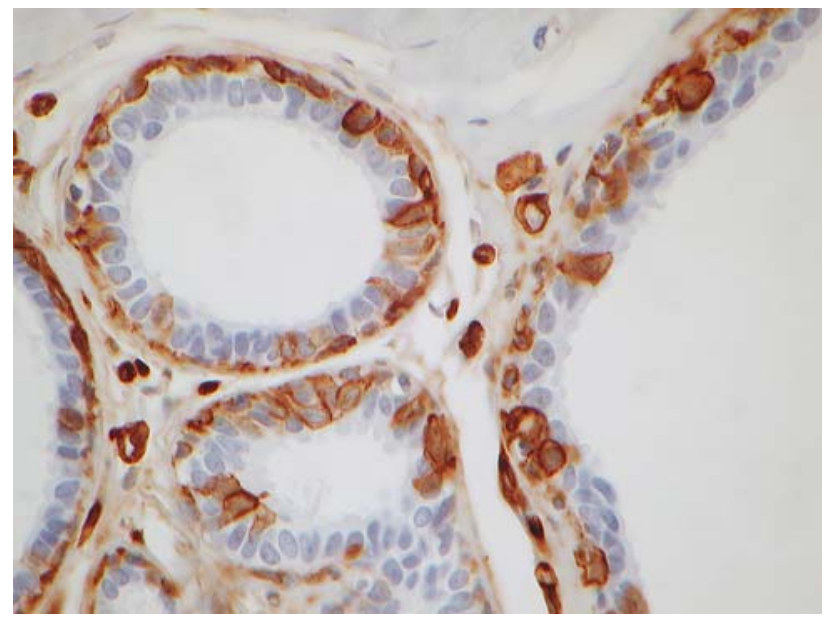

Fig. 3 AQP1 expression in the terminal ductulo-lobular unit is detected in myoepithelial cells, capillary endothelial cells and a few luminal epithelial cells

hyperplasia, a cytokeratin 5/6-like mosaic pattern of AQP1 staining was noticed. As a general rule, endothelial cells of small arteries and lymphatic vessels were not stained by anti-AQP1. Smooth muscle cells, even the myocytes of arterial vessel walls, displayed a diffuse granular cytoplasmic staining without membranous staining. No positive nuclear immunoreaction was observed.

\section{Relationship between AQP1 expression and established clinico-pathological parameters}

Frequency distribution of established clinico-pathological parameters and their relation to AQP1 expression is summarized in Table 1 . The mean age of the patients was 53 years (range 26-85 years.) The majority of tumours (73.4\%) were invasive ductal carcinomas and $18.2 \%$ were of invasive lobular type; all other tumour types were rare

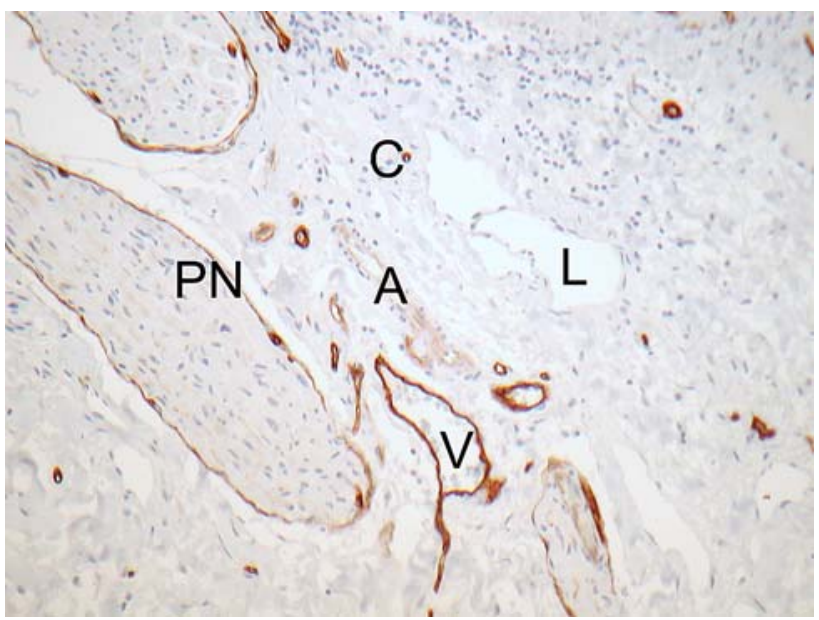

Fig. 4 AQP1 antibody stains perineural sheets $(P N)$ as well as capillary $(C)$ and venous $(V)$ endothelial cells, but not the endothelia of arterioles $(A)$ and lymphatic vessels $(L)$. Only the muscle cells of arterioles show a weak granular cytoplasmic staining pattern

$(<4 \%)$. Three-quarters of the carcinomas were $>16 \mathrm{~mm}$ and $53.2 \%$ had lymph node metastases at the time of diagnosis. $87 \%$ of the tumours were classified as grade 2 or grade 3; the Nottingham-Prognostic index showed a well balanced distribution of its categories ranging from 26 to $38 \%$. About $70 \%$ of carcinomas were ER-positive and $90 \%$ HER2 negative. The CK14-positive basal-like immunophenotype was present in 18 (8.9\%) of the cases.

All AQP1 positive invasive carcinomas were of ductal type, estrogen receptor negative and HER2/neu negative. All markers and marker combinations-CK14, SMA, triple-negativity and medullary-like histology-were highly significantly related to AQP1 expression (Pearson's $\chi^{2}$ ranged from 2.173 to $5.869, p<0.000$ for each). Eight of the 11 AQP1 positive cases showed co-expression of CK14, but all AQP1 positive cases revealed a positive immunostaining with at least one of the other investigated basal-like markers, as well.

AQP1 expression showed significant direct correlation with tumour grade (Pearson's $r=0.139, p=0.049$ ), medullary-like histology (Pearson's $r=0.327, p<0.000$ ), triple-negativity (Pearson's $r=0.443 ; p<0.000$ ), CK14 and SMA expression (Pearson's $r=0.538$ and 0.364 , respectively, $p<0.000$ for both), whereas, it had highly significant negative correlation with ER status (Pearson's $r=-0.378, p<0.000)$.

Uni- and multivariate long-term survival analysis

The possible impact of patients, tumour variables and treatment modalities was investigated by univariate analysis with respect to overall survival (Table 2). The highest prognostic value was associated with the NPI, followed by 
Table 1 Frequency and distribution of AQP1 expression in association with established clinico-pathological parameters, investigational molecular markers and therapy

\begin{tabular}{|c|c|c|c|c|}
\hline & & All patients $n(\%)$ & AQP1 positive $n(\%)$ & AQP1 negative $n(\%)$ \\
\hline Number of patients & & $203(100)$ & $11(5.4)$ & $92(94.6)$ \\
\hline \multirow[t]{2}{*}{ Age } & $26-53$ & 85 (41.9) & $4(4.7)$ & $81(95.3)$ \\
\hline & $54-85$ & $118(58.1)$ & $7(5.9)$ & $111(94.1)$ \\
\hline \multirow[t]{8}{*}{ Tumour type } & Ductal & $149(73.4)$ & $11(7.4)$ & $138(92.6)$ \\
\hline & Lobular & $37(18.2)$ & $0(0)$ & 37 (100) \\
\hline & Mucinous & $5(2.5)$ & $0(0)$ & $5(100)$ \\
\hline & Apocrine & $8(3.9)$ & $0(0)$ & $8(100)$ \\
\hline & Tubular & $1(0.5)$ & $0(0)$ & $1(100)$ \\
\hline & Micropapillary & $1(0.5)$ & $0(0)$ & $1(100)$ \\
\hline & Glycogen-rich & $1(0.5)$ & $0(0)$ & $1(100)$ \\
\hline & Neuroendocrine & $1(0.5)$ & $0(0)$ & $1(100)$ \\
\hline \multirow[t]{2}{*}{ Tumour size } & $\leq 15 \mathrm{~mm}$ & $50(24.6)$ & $2(4.0)$ & $48(96.0)$ \\
\hline & $>15 \mathrm{~mm}$ & $153(75.4)$ & $9(5.9)$ & $144(94.1)$ \\
\hline \multirow[t]{2}{*}{ Lymph node status } & Negative & $95(46.8)$ & $6(6.3)$ & $89(93.7)$ \\
\hline & Positive & $108(53.2)$ & $5(4.6)$ & $103(95.4)$ \\
\hline \multirow[t]{3}{*}{ Histological grade } & 1 & $24(11.8)$ & $0(0)$ & $24(100)$ \\
\hline & 2 & 87 (42.9) & $3(3.4)$ & $84(96.6)$ \\
\hline & 3 & $92(45.3)$ & $8(8.7)$ & $84(91.3)$ \\
\hline \multirow[t]{3}{*}{ NPI } & Good $(\leq 3.4)$ & $76(37.6)$ & $2(2.6)$ & $74(97.4)$ \\
\hline & Moderate (3.41-5.4) & $73(36.1)$ & $4(5.6)$ & $69(94.5)$ \\
\hline & Poor $(>5.4)$ & $53(26.2)$ & $5(9.4)$ & $8(90.6)$ \\
\hline \multirow[t]{2}{*}{ ER } & Negative & $58(28.6)$ & $11(19.0)$ & $47(81.0)$ \\
\hline & Positive & $145(71.4)$ & $0(0)$ & $145(100)$ \\
\hline \multirow[t]{2}{*}{ PR } & Negative & 78 (40.6) & $10(12.8)$ & $68(87.2)$ \\
\hline & Positive & $114(59.4)$ & $1(0.9)$ & $113(99.1)$ \\
\hline \multirow[t]{2}{*}{ HER2/neu } & Negative & $181(89.6)$ & $11(6.1)$ & $170(93.9)$ \\
\hline & Positive (3+) & $21(10.4)$ & $0(0)$ & $21(100)$ \\
\hline \multirow[t]{2}{*}{ CK14 } & Negative & $185(91.1)$ & $3(1.6)$ & $182(98.4)$ \\
\hline & Positive & $18(8.9)$ & $8(44.4)$ & $10(55.6)$ \\
\hline SMA & Negative & $189(93.1)$ & $6(3.2)$ & $183(96.8)$ \\
\hline (Smooth-muscle actin) & Positive & $14(6.9)$ & $5(35.7)$ & $9(64.3)$ \\
\hline Triple-negativity & Non triple-negative & 157 (77.7) & $1(0.6)$ & $156(99.4)$ \\
\hline (ER-, PR- HER2-) & Triple negative & $45(22.3)$ & $10(22.2)$ & $35(77.8)$ \\
\hline \multirow[t]{2}{*}{ Medullary-like histology } & Negative & $193(95.1)$ & $7(3.6)$ & $186(96.4)$ \\
\hline & Positive & $10(4.9)$ & $4(40.0)$ & $6(60.0)$ \\
\hline \multirow[t]{2}{*}{ Surgery } & Breast conserving & $38(18.7)$ & $3(7.9)$ & $35(92.1)$ \\
\hline & Ablative & $165(81.3)$ & $8(4.8)$ & $157(95.2)$ \\
\hline \multirow[t]{2}{*}{ Tamoxifen } & No & $140(69.0)$ & $9(6.4)$ & $131(93.6)$ \\
\hline & Yes & $63(31.0)$ & $2(3.2)$ & $61(96.8)$ \\
\hline \multirow[t]{2}{*}{ CMF } & No & $140(69.0)$ & $9(6.4)$ & $131(93.6)$ \\
\hline & Yes & $63(31.0)$ & $2(3.2)$ & $61(96.8)$ \\
\hline \multirow[t]{2}{*}{ Tamoxifen + CMF } & No & 194 (95.6) & $11(5.7)$ & $183(94.3)$ \\
\hline & Yes & $9(4.4)$ & $0(0)$ & $9(4.7)$ \\
\hline \multirow[t]{2}{*}{ Radiation } & No & $156(76.8)$ & $8(5.1)$ & 148 (94.9) \\
\hline & Yes & $47(23.2)$ & $3(6.4)$ & 44 (93.6) \\
\hline
\end{tabular}


Table 2 Association between the clinicopathological prognostic parameters and overall survival in univariate analysis

\begin{tabular}{lrl}
\hline & Log rank & $p$ \\
\hline Age & 5.511 & 0.019 \\
Tumour type & 2.888 & NS \\
Tumour size & 8.321 & 0.004 \\
Lymph node status & 28.626 & 0.000 \\
Histological grade & 16.389 & 0.000 \\
NPI-Group & 46.675 & 0.000 \\
ER & 8.023 & 0.005 \\
HER2/neu & 0.055 & NS \\
Triple negativity & 12.706 & 0.000 \\
Medullary-like histology & 10.465 & 0.001 \\
CK14 & 9.454 & 0.002 \\
SMA & 4.872 & 0.027 \\
AQP1 & 15.400 & 0.000 \\
Surgical treatment & 2.776 & NS \\
Tamoxifen & 3.959 & NS \\
CMF & 4.090 & 0.047 \\
Radiation & 1.892 & NS \\
\hline NS & &
\end{tabular}

NS not significant

lymph node status, and histopathological grade. AQP1 expression was also highly significantly associated with a shorter cancer-specific survival (log rank chi-square $=$ 15.400; $p<0.000$; Fig. 5). All other established prognostic markers, such as age, tumour size, ER status, as well as markers of basal-like carcinomas were significantly associated with overall survival as well, whereas, tumour type and HER2 status did not achieve significance. Of the treatment modalities used, only adjuvant CMF therapy revealed a significant association with overall survival in the univariate analysis ( $\log$ rank chi-square $=3.959 ; p<0.047$ ).

In the multivariate Cox regression analysis (Table 3), including all cases (Model 1, $n=203$ ), lymph node status and age were independent prognostic markers. AQP1 expression achieved high independent prognostic significance after further stratification of data and exclusion of the other basal-like markers (medullary-like histology, CK14 and SMA; Model 2). In the high grade subgroup, (Model 3, $n=92$, data not shown), including age, pT, pN, ER, medullary-like histology, CK14, SMA, AQP1 and CMF therapy, only the presence of lymph node metastases $(p=0.005)$ and AQP1 expression $(p=0.030)$ were independent predictors of poor survival. In the ER negative subgroup, (Model 4, $n=58$, data not shown), including age, pT, pN, grade, medullary-like histology, CK14, SMA, AQP1 and CMF therapy, only age $(p=0.032)$, CMF therapy $(p=0.008)$ and AQP1 expression $(p=0.016)$ were independent prognostic factors. In the

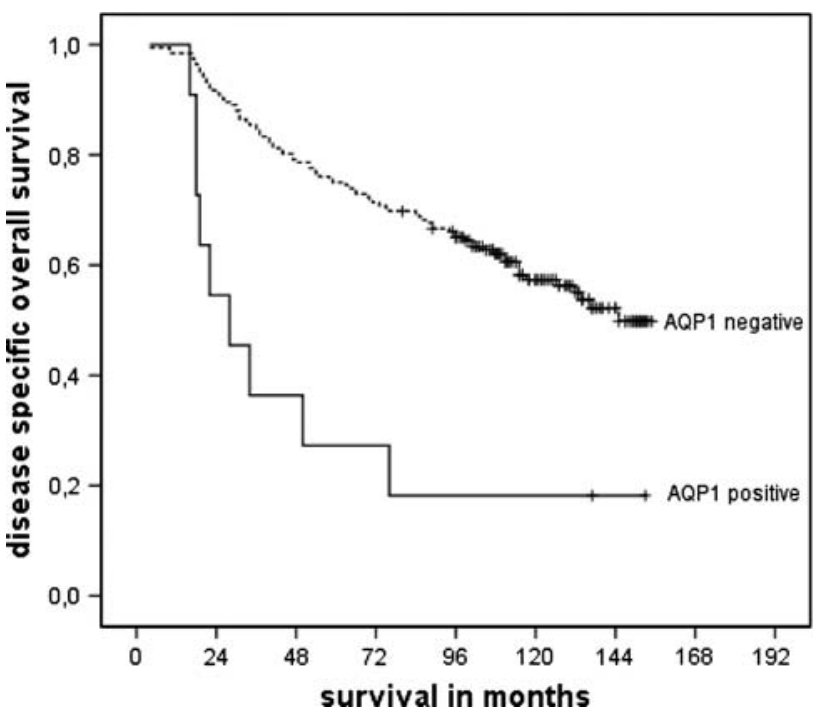

Fig. 5 Cumulative survival of patients with sporadic invasive breast cancer in correlation to AQP1 expression (Kaplan-Meier analysis, log rank test, $p=0.000$ )

subgroup of nodal-negative patients (Model 5, $n=95$ ), age $(p=0.025)$ and AQP1 expression $(p=0.001)$ were the only independent prognostic markers, whereas, in the nodal-positive subgroup (Model 6; $n=108$ ), only CK14 expression $(p=0.010)$ predicted poor outcome independently.

\section{Discussion}

The present study demonstrated that AQP1 expression is significantly associated with the basal-like phenotype of breast carcinomas and poor survival of the patients. In multivariate analysis, AQP1 expression proved to be the strongest marker of poor prognosis among all other basallike parameters (CK14, SMA, triple negativity for ER, PR and Her2neu, as well as medullary like histology) studied: AQP1 achieved a high independent level of significance for the total cohort of patients studied after the exclusion of the other basal-like markers from the analysis. In the subgroup analysis, AQP1 expression was a highly significant independent marker of poor prognosis in the high-grade, ER-negative and nodal-negative subgroups of patients (Table 3).

The concept of basal-like carcinoma (BLBC) of the breast forming a distinct molecular subtype of invasive breast cancer with aggressive biological behaviour has only recently emerged $[17,18]$. BLBCs constitute $2-18 \%$ of all breast cancer [15] and are characterised by high histological grade, lack of hormone receptors and HER2 expression $[13,19]$ and show some characteristics of normal breast basal/myoepithelial cells [20]. In the present study, the 
Table 3 Multivariate cox regression analysis of breast cancer specific survival (only representative stratifications shown)

\begin{tabular}{|c|c|c|c|}
\hline & \multicolumn{3}{|l|}{ Death } \\
\hline & Hazard ratio & $95 \% \mathrm{CI}$ & $p$ \\
\hline \multicolumn{4}{|l|}{ Model 1 (all cases; $n=203$ ) } \\
\hline Age $>54$ & 1.777 & $1.066-2.962$ & 0.028 \\
\hline Tumour size $>15 \mathrm{~mm}$ & 1.630 & $0.898-2.959$ & NS \\
\hline Lymph node positive & 2.880 & $1.619-5.125$ & 0.000 \\
\hline Histological grade (G2) & 0.601 & $0.221-1.635$ & NS \\
\hline Histological grade (G3) & 0.795 & $0.496-1.275$ & NS \\
\hline ER negative & 0.675 & $0.398-1.146$ & NS \\
\hline Medullary-like histology & 1.534 & $0.668-3.521$ & NS \\
\hline CK14 positive & 1.068 & $0.322-3.544$ & NS \\
\hline SMA positive & 1.062 & $0.376-2.996$ & NS \\
\hline AQP1 positive & 2.854 & $0.952-8.557$ & 0.061 \\
\hline $\mathrm{CMF}$ & 1.092 & $0.630-1.893$ & NS \\
\hline \multicolumn{4}{|c|}{ Model 2 (all cases; CK14, SMA and medullary-like histology excluded) } \\
\hline Age $>54$ & 1.771 & $1.064-2.947$ & 0.028 \\
\hline Tumour size $>15 \mathrm{~mm}$ & 1.679 & $0.929-3.035$ & NS \\
\hline Lymph node positive & 2.893 & $1.634-5.112$ & 0.000 \\
\hline Histological grade (G2) & 0.591 & $0.218-1.604$ & NS \\
\hline Histological grade (G3) & 0.767 & $0.482-1.219$ & NS \\
\hline ER negative & 0.632 & $0.383-1.043$ & NS \\
\hline AQP1 positive & 3.423 & $1.557-7.523$ & 0.002 \\
\hline CMF & 1.062 & $0.618-1.823$ & NS \\
\hline \multicolumn{4}{|c|}{ Model 5 (node-negative subgroup, $n=95$ ) } \\
\hline Age $>54$ & 3.244 & $1.156-9.103$ & 0.025 \\
\hline Tumour size $>15 \mathrm{~mm}$ & 1.435 & $0.521-3.953$ & NS \\
\hline Histological grade (G2) & 0.796 & $0.216-2.941$ & NS \\
\hline Histological grade (G3) & 0.750 & $0.250-2.249$ & NS \\
\hline ER negative & 0.553 & $0.187-1.634$ & NS \\
\hline Medullary-like histology & 2.188 & $0.509-9.420$ & NS \\
\hline CK14 positive & 0.531 & $0.083-3.387$ & NS \\
\hline SMA positive & 0.161 & $0.012-2.100$ & NS \\
\hline AQP1 positive & 15.721 & $3.221-76.721$ & 0.001 \\
\hline $\mathrm{CMF}$ & 1.877 & $0.214-16.495$ & NS \\
\hline \multicolumn{4}{|c|}{ Model 6 (node-positive subgroup, $n=108$ ) } \\
\hline Age $>54$ & 1.207 & $0.570-2.555$ & NS \\
\hline Tumour size $>15 \mathrm{~mm}$ & 1.691 & $0.783-3.652$ & NS \\
\hline Histological grade (G2) & 0.000 & $0.000-1.755 \mathrm{E} 274$ & NS \\
\hline Histological grade (G3) & 0.754 & $0.438-1.301$ & NS \\
\hline ER negative & 0.852 & $0.448-1.621$ & NS \\
\hline Medullary-like histology & 0.566 & $0.163-1.970$ & NS \\
\hline CK14 positive & 7.892 & $1.625-38.321$ & 0.010 \\
\hline SMA positive & 1.470 & $0.430-5.025$ & NS \\
\hline AQP1 positive & 0.650 & $0.160-2.643$ & NS \\
\hline CMF & 0.696 & $0.347-1.397$ & NS \\
\hline
\end{tabular}

NS not significant 
immunophenotypic identification of malignant basal-like epithelial cells relied on the detection of CK14 positivity, a type I cytokeratin which usually forms a heterotetramer with keratin 5, a type II keratin. Together, they form the cytoskeleton of normal basal epithelial and myoepithelial cells. CK14 has also been used as a marker of basal mammary epithelial cells with in vivo regenerative ability in searching for mammary gland progenitor and stem cells [21] Further, basal-like carcinomas consistently overexpress EGFR, show the highest proliferation rates, and are associated with poor clinical outcomes [22]. In addition, they have also been described as the prevalent subtype in BRCA1-related breast carcinomas [23, 24].

Despite the increasing number of publications focusing on the morphological and immunohistochemical characterization of this entity, there is no international consensus yet on the complement of biomarkers or pathologic features that precisely defines BLBC. Furthermore, there is a growing body of evidence indicating that BLBCs are heterogeneous and encompass tumours with distinct biological and morphological characteristics [16].

According to our results, AQP1 expression apparently represents a novel biomarker for a particularly aggressive subgroup of breast carcinomas with basal-like phenotype. AQP1 was only detected in a subset (73\%) of CK14 positive BLBCs, especially in the subgroups of patients with high grade and nodal negative carcinomas, who experienced particularly short overall survival.

In the literature, the prognostic significance of basal-like features is still controversial discussed and decreased overall survival can only be predicted if the tumour relapses within the first 5 years of follow-up [25]. In our cohort, not influenced by modern chemotherapy regiments, all of the tested basal like parameters were significantly associated with decreased overall survival in univariate analyses, indicating a very aggressive behaviour of BLBC's. Moreover, there is seemingly a paradoxical dissociation between biological aggressiveness and chemosensitivity in BLBC. In spite of the low differentiation grade of the tumours, BLBCs seem to be more responsive to neoadjuvant anthracyclin-based chemotherapy than other ductal carcinomas with luminal subtypes [26].

Due to the time period (1989-1993) of the present series, our results were not influenced by data of patients treated with such adjuvant chemotherapy regime. In our cohort studied, only CMF treatment was associated with poor overall survival in univariate analysis, which might be a consequence of the application of adjuvant chemotherapy only in high risk cases. AQP1 expression was not correlated with any of the therapy modalities.

Nevertheless, our results might have been slightly biased by the complete lack of salivary gland-like, metaplastic and myoepithelial carcinomas within this series, which are supposed to represent a part of the BLBC spectrum [16, 27-31], although some of these rather rare carcinoma types are associated with a more favourable prognosis [8].

It has been recently proposed that BLBC is endowed with stem/progenitor cell features. It has been suggested that a CK5/14-positive breast progenitor cell able to differentiate into both luminal and myoepithelial cells of the normal breast would be the initially transformed cell in basal-phenotype breast cancer [32, 33]. Most recent experimental results confirmed that BLBCs indeed express high levels of stem cell regulatory genes, i. e. CD133, Bmil and SLUG [34], the latter correlating with the acquisition of a migrant phenotype in a variety of cell types [35] and particularly metastatic breast cancer [36]. AQP1 expression has also been found to increase tumour cell migration and spread in melanoma and breast cancer cell lines in vivo, suggesting a novel function of AQP1 in highgrade tumours [5]. The histological counterpart of these particularly aggressive tumour cells may be the ones exhibiting the undifferentiated basal/stem cell-like phenotype in a subgroup of BLBCs.

The biological process of epithelial differentiation may reflect the ductal or basal-phenotype tumour origin. In keeping with the concept of breast carcinogenesis from a putative cancer stem cell, ductal carcinomas would arise from cancer stem/progenitor cells, which have retained their capacity to differentiate, whereas, basal-like breast carcinomas may represent tumours in which the lineage commitment process of stem cells has been arrested [37]. Since the basal-like phenotype is overrepresented in patients with BRCA1 mutations, the association and possible biological significance of AQP1 and BRCA1 has to be further elucidated in hereditary breast cancer cases [23].

Regarding tissue- and cell-type-specific distribution of AQP1 in tumour-adjacent normal-appearing breast tissues, AQP1 was mainly located in myoepithelial cells of ducts and lobules, endothelial cells of capillary and venous blood vessels, but not in arteries and lymphatics, smooth muscle cells of vessel walls and perineurial sheets of peripheral nerve fibres. The presence of a few luminal epithelial cells in normal lobules expressing AQP1 may indicate that $\mathrm{AQP} 1$ expression is a further characteristic of a postulated CK14 positive progenitor cell in the breast.

Although not studied systematically, the consistent finding of exclusive AQP1 expression only in capillary and venous endothelial cells, but not in arterial and lymphatic endothelial cells indicate a biological and functional difference in these endothelial compartments. Like other basal and myoepithelial markers, AQP1 antibodies may prove to be helpful in the differential diagnosis of benign, preneoplastic and pre-invasive breast lesions in routine pathology but the staining patterns in these lesions remain to be studied in detail [38]. 
AQP1 expression and distribution in normal and various human tumour TMAs by immunohistochemistry have been recently described by Mobasheri and co-workers [3], who suggested that AQP1 could be considered as a marker of microvasculature; its increased expression in some human adenocarcinomas may thus be due to angiogenesis. Additionally, Endo et al. [39] described a heterogeneous expression of AQP1 in tumour cells and their vasculature in xenotransplanted mammary carcinomas and glioblastomas in vivo. These results together with findings of an increased migration and metastatic potential of AQP1-expressing cancer cell lines indicate an important role of AQP1 in tumour spread of some highly aggressive human cancers [5]. AQP1-dependent cell migration was shown to be related to polarized expression of ion transporters and AQP1 at the leading edge of migrating cells. Their parallel activity creates an osmotic gradient, which drives the influx of water across the cell membrane. The increased local hydrostatic pressure results in membrane protrusions (lamellipodia), which in turn create place for actin depolymerisation. The coexpression of AQP1, SMA and CK14 may support the importance of a cross-talk of AQP1, contractile filaments and cytoskeleton filaments for cell remodelling in cell migration. Thus, manipulation of the expression or function of tumour AQPs may alter their invasiveness and metastatic potential [40]. The expression of AQP1 in breast carcinomas may provide a therapeutic target both as a cell surface marker and for functional intervention. Inhibition of AQP1 expression, for example by siRNA, or AQP1 function (with a blocking antibody or a small inhibitory molecule) may result in a reduced invasive potential of breast carcinoma cells [7, 41].

Another potential way to interfere with AQP1-induced tumour growth and spread seems to be the inhibition of angiogenesis. Studies based on acetazolamide and topiramate (carbonic anhydrase inhibitors reducing cancer invasiveness in vitro), indicated a significant correlation between angiogenesis inhibition and suppression of AQP1 gene in an experimental mouse model of Lewis lung carcinoma [42, 43]. These studies associated the decreased AQP1 expression induced by these drugs with the reduction of tumour metastases, probably due to the reduction of the number of microvessels.

Detection of new biomarkers for an effective targeted anti-tumour therapy is an outstanding challenge in breast cancer research particularly for the relatively small subset of hormone- and HER2 negative breast carcinomas with poor clinical outcome despite initial chemosensitivity to anthracyclin-based neoadjuvant chemotherapy. Most of these carcinomas exhibit a basal-like phenotype. This work provides evidence for the first time in a clinico-pathological setting that AQP1 may identify a small subset of patients with poorer-prognosis basal-like breast carcinomas-findings which should be further explored and verified.
Acknowledgments The technical support by N. Cramer, S. Ladwig, B. Linker and M. F. Saballs is gratefully acknowledged.

\section{References}

1. Denker BM, Smith BL, Kuhajda FP et al (1988) Identification, purification, and partial characterization of a novel Mr 28, 000 integral membrane protein from erythrocytes and renal tubules. J Biol Chem 263:15634-15642

2. Matsuzaki T, Suzuki T, Koyama H et al (1999) Water channel protein AQP3 is present in epithelia exposed to the environment of possible water loss. J Histochem Cytochem 47:1275-1286

3. Mobasheri A, Marples D (2004) Expression of the AQP-1 water channel in normal human tissues: a semiquantitative study using tissue microarray technology. Am J Physiol Cell Physiol 286:529-537. doi:10.1152/ajpcell.00408.2003

4. Magni F, Sarto C, Ticozzi D et al (2006) Proteomic knowledge of human aquaporins. Proteomics 6:5637-5649. doi:10.1002/pmic. 200600212

5. Hu J, Verkman AS (2006) Increased migration and metastatic potential of tumor cells expressing aquaporin water channels. FASEB J 20:1892-1894. doi:10.1096/fj.06-5930fje

6. Lanahan A, Williams JB, Landers LK et al (1992) Growth factorinduced delayed early response genes. Mol Cell Biol 12:39193929

7. Saadoun S, Papadopoulos MC, Hara-Chikuma M et al (2005) Impairment of angiogenesis and cell migration by targeted aquaporin-1 gene disruption. Nature 434:786-792. doi:10.1038/ nature 03460

8. Ellis IO, Schnitt SJ, Sastre-Garau X (2003) Invasive breast carcinoma. In: Tavassoli FA, Devilee P et al (eds) Pathology and genetics of tumours of the breast and female genital organs. IARC Press, Lyon, pp 21-22

9. Sobin LH, Wittekind CH (2002) UICC: TNM classification of malignant tumors, 6th edn. Wiley-Liss, New York

10. Elston CW, Ellis IO (1991) Pathological prognostic factors in breast cancer. I. The value of histological grade in breast cancer: experience from a large study with long-term follow-up. Histopathology 19:403-410. doi:10.1111/j.1365-2559.1991.tb00229.x

11. Galea MH, Blamey RW, Elston CE et al (1992) The Nottingham prognostic index in primary breast cancer. Breast Cancer Res Treat 22:207-219. doi:10.1007/BF01840834

12. McShane LM, Altman DG, Sauerbrei W et al (2005) Reporting recommendations for tumor marker prognostic studies. J Clin Oncol 23:9067-9072. doi:10.1200/JCO.2004.01.0454

13. Fadare O, Tavassoli FA (2007) The phenotypic spectrum of basal-like breast cancers: a critical appraisal. Adv Anat Pathol 14:358-373. doi:10.1097/PAP.0b013e31814b26fe

14. Laakso M, Loman N, Borg A et al (2005) Cytokeratin 5/14-positive breast cancer: true basal phenotype confined to BRCA1tumors. Mod Pathol 18:1321-1328. doi:10.1038/modpathol.3800456

15. Banerjee S, Reis-Filho JS, Ashley S et al (2006) Basal-like breast carcinomas: clinical outcome and response to chemotherapy. J Clin Pathol 59:729-735. doi:10.1136/jcp.2005.033043

16. Rakha EA, Putti TC, Abd El-Rehim DM et al (2006) Morphological and immunophenotypic analysis of breast carcinomas with basal and myoepithelial differentiation. J Pathol 208:495506. doi:10.1002/path.1916

17. Perou CM, Sørlie T, Eisen MB et al (2000) Molecular portraits of human breast tumours. Nature 17(406):747-752. doi:10.1038/ 35021093

18. Van 't Veer LJ, Dai H, van de Vijver MJ et al (2002) Gene expression profiling predicts clinical outcome of breast cancer. Nature 415:530-536. doi:10.1038/415530a 
19. Mani SA, Yang J, Brooks M et al (2007) Mesenchyme forkhead 1 (FOXC2) plays a key role in metastasis and is associated with aggressive basal-like breast cancers. Proc Natl Acad Sci USA 104:10069-10074. doi:10.1073/pnas.0703900104

20. Nielsen TO, Hsu FD, Jensen K et al (2004) Immunohistochemical and clinical characterization of the basal-like subtype of invasive breast carcinoma. Clin Cancer Res 10:5367-5374. doi:10.1158/ 1078-0432.CCR-04-0220

21. Eirew P, Stingl J, Raouf A et al (2008) A method for quantifying normal human mammary epithelial stem cells with in vivo regenerative ability. Nat Med 14:1384-1389. doi:10.1038/nm.1791

22. Sorlie T, Perou CM, Tibshirani R et al (2001) Gene expression patterns of breast carcinomas distinguish tumor subclasses with clinical implications. Proc Natl Acad Sci USA 98:10869-10874. doi:10.1073/pnas.191367098

23. Foulkes WD, Stefansson IM, Chappuis PO et al (2003) Germline BRCA1 mutations and a basal epithelial phenotype in breast cancer. J Natl Cancer Inst 95:1482-1485

24. Rodríguez-Pinilla SM, Rodríguez-Gil Y, Moreno-Bueno G et al (2007) Sporadic invasive breast carcinomas with medullary features display a basal-like phenotype: an immunohistochemical and gene amplification study. Am J Surg Pathol 31:501-508. doi:10.1097/01.pas.0000213427.84245.92

25. Da Silva L, Clarke C, Lakhani SR (2007) Demystifying basal-like breast carcinomas. J Clin Pathol 60:1328-1332. doi:10.1136/jcp. 2006.041731

26. Carey LA, Dees EC, Sawyer L et al (2007) The triple negative paradox: primary tumor chemosensitivity of breast cancer subtypes. Clin Cancer Res 13:2329-2334. doi:10.1158/1078-0432. CCR-06-1109

27. Livasy CA, Karaca G, Nanda R et al (2006) Phenotypic evaluation of the basal-like subtype of invasive breast carcinoma. Mod Pathol 19:264-271. doi:10.1038/modpathol.3800528

28. Reis-Filho JS, Milanezi F, Steele D et al (2006) Metaplastic breast carcinomas are basal-like tumours. Histopathology 49:10-21. doi:10.1111/j.1365-2559.2006.02467.x

29. Fulford LG, Easton DF, Reis-Filho JS et al (2006) Specific morphological features predictive for the basal phenotype in grade 3 invasive ductal carcinoma of breast. Histopathology 49:22-34. doi:10.1111/j.1365-2559.2006.02453.x

30. Jacquemier J, Padovani L, Rabayrol L et al (2005) Typical medullary breast carcinomas have a basal/myoepithelial phenotype. J Pathol 207:260-268. doi:10.1002/path.1845

31. Tsuda H, Takarabe T, Hasegawa F et al (2000) Large, central acellular zones indicating myoepithelial tumor differentiation in high-grade invasive ductal carcinomas as markers of predisposition to lung and brain metastases. Am J Surg Pathol 24:197202. doi:10.1097/00000478-200002000-00005

32. Boecker W, Moll R, Poremba C et al (2002) Common adult stem cells in the human breast give rise to glandular and myoepithelial cell lineages: a new cell biological concept. Lab Invest 82:737745

33. Boecker W, Buerger H (2003) Evidence of progenitor cells of glandular and myoepithelial cell lineages in the human adult female breast epithelium: a new progenitor (adult stem) cell concept. Cell Prolif 36:73-84. doi:10.1046/j.1365-2184.36.s.1.7.x

34. Storci G, Sansone P, Trere D et al (2008) The basal-like breast carcinoma phenotype is regulated by SLUG gene expression. J Pathol 214:25-37. doi:10.1002/path.2254

35. Barrallo-Gimeno A, Nieto MA (2005) The snail genes as inducers of cell movement and survival: implications in development and cancer. Development 132:3151-3161. doi:10.1242/dev.01907

36. Côme C, Magnino F, Bibeau F et al (2006) Snail and slug play distinct roles during breast carcinoma progression. Clin Cancer Res 12:5395-5402. doi:10.1158/1078-0432.CCR-06-0478

37. Al-Hajj M, Wicha MS, Benito-Hernandez A et al (2003) Prospective identification of tumorigenic breast cancer cells. Proc Natl Acad Sci USA 100:3983-9388. doi:10.1073/pnas.053029 1100

38. Otterbach F, Bànkfalvi A, Bergner S et al (2000) Cytokeratin 5/6 immunohistochemistry assists the differential diagnosis of atypical proliferations of the breast. Histopathology 37:232-240. doi:10.1046/j.1365-2559.2000.00882.x

39. Endo M, Jain RK, Witwer B et al (1999) Water channel (aquaporin 1) expression and distribution in mammary carcinomas and glioblastomas. Microvasc Res 58:89-98. doi:10.1006/mvre. 1999.2158

40. Schwab A, Nechyporuk-Zloy V, Fabian A et al (2007) Cells move when ions and water flow. Pflugers Arch 453:421-432. doi: 10.1007/s00424-006-0138-6

41. Moon C, King LS, Agre P (1997) Aqp1 expression in erythroleukemia cells: genetic regulation of glucocorticoid and chemical induction. Am J Physiol 273:C1562-C1570

42. Xiang Y, Ma B, Li T et al (2004) Acetazolamide inhibits aquaporin-1 protein expression and angiogenesis. Acta Pharmacol Sin 25:812-816

43. Ma B, Xiang Y, Li T et al (2004) Inhibitory effect of topiramate on Lewis lung carcinoma metastasis and its relation with AQP1 water channel. Acta Pharmacol Sin 25:54-60 